\title{
Trachoma in the Gambia
}

\author{
P J Dolin, H Faal, G J Johnson, J Ajewole, A A Mohamed, Pak S Lee
}

\begin{abstract}
Background-As part of the second National Survey of Blindness and Low Vision in the Gambia carried out in 1996, all survey participants were examined for signs of trachoma. The findings were compared with the results of the first survey in 1986, which used the same sampling strategy.

Methods-A multistage stratified cluster random sample, with proportional probability sampling, was obtained. Stratification included settlement size (less than 400 residents, and 400 and more residents). All subjects were examined for trachoma using the simplified WHO grading system. Results-Of the sample of 14110 people, $13047(92.5 \%)$ were examined. Active inflammatory trachoma (grade TF or TI) was found in $3.0 \%$ of all age groups and $5.9 \%$ of children aged 0-9 years old. Trichiasis was found in $3.3 \%$ and trachomatous corneal opacities in $0.9 \%$ of adults aged 30 and over. The prevalence of blinding trachomatous corneal opacities was $0.02 \%$, compared with $0.10 \% 10$ years previously.

Conclusion-Compared with a previous national survey undertaken in 1986, prevalence of active trachoma has fallen by $54 \%$. There has been an $80 \%$ relative reduction in blinding trachomatous corneal opacities over the $\mathbf{1 0}$ year period. (Br F Ophthalmol 1998;82:930-933)
\end{abstract}

The Gambia is a small country in west Africa with a population of 1.2 million. Trachoma was mesoendemic in the Gambia until recently and corneal opacities from trachoma were a leading cause of blindness in the population. As part of the second National Survey of Blindness and Low Vision carried out in 1996, all survey participants were examined for signs of trachoma. In this paper a description of the current epidemiology of trachoma in the Gambia is presented, and comparisons are made with findings from the first National Survey of Blindness and Low Vision, which was undertaken in 1986.

National Eye Care

Programme, Ministry of Health, the Gambia H Faal

J Ajewole

Correspondence to: Dr Paul Dolin, Global Tuberculosis Programme, World Health Organisation, 1211 Geneva 27,

Switzerland

Accepted for publication 4 March 1998 \begin{abstract}
seven divisions and within each division a sample of districts was selected and, within each selected district, a sample of census enumera-

A dethods methods will be presented elsewhere. ${ }^{2}$ Briefly, multistage stratified cluster random sampling, with proportional probability sampling, was used to select a cross sectional sample of the
\end{abstract}

tion areas was selected and, within each enumeration area a sample of compounds was selected. All residents from the selected compounds were included in the sample. This was the same sampling strategy as employed in the 1986 survey. A census enumeration area is a small census population unit, averaging around 500 people. The sample of census enumeration areas was selected using proportional probability sampling, with stratification by settlement size (small, fewer than 400 residents; large, 400 and more residents). An enumeration area is not equivalent to a village, but typically covers one or more small settlements or part of a larger settlement.

Using this method, 73 settlements were randomly selected yielding a sample of 14110 people, of whom 13047 (92.5\%) were examined.

The presence of trachoma was determined by examination using a binocular $2.5 \times$ loupe and a focusing torch. Examinations were undertaken and trachoma was graded using the simplified WHO system. ${ }^{3}$ The following five grades were used: $\mathrm{TF}$ (trachomatous inflammation, follicular), presence of five or more trachomatous follicles in the central area of the upper tarsal conjunctiva; TI (trachomatous inflammation, intense), pronounced inflammatory thickening of the upper tarsal conjunctiva that obscures more than half of the normal deep tarsal vessels; TS (trachomatous scarring), presence of scarring in the tarsal conjunctiva; TT (trachomatous trichiasis) at least one eyelash rubbing on the eyeball; CO (corneal opacity) easily visible corneal opacity obscuring the pupil. ${ }^{3}$ Each examiner received training on the identification and grading of trachoma. A series of interobserver studies were undertaken to ensure that each examiner was correctly grading trachoma with minimal or no variation between examiners.

The relation between active infection with Chlamydia trachomatis and signs of inflammatory trachoma is not straightforward. ${ }^{4}$ Using extremely sensitive polymerase chain reaction based assays, there was a proportion of subjects with unequivocal signs of active trachoma in whom the agent was not detectable. Conversely, chlamydial DNA was found in a proportion of subjects without evidence of inflammatory trachoma as defined by $\mathrm{TF}$ or TI. The development and resolution of clinical signs seems to lag behind the appearance and disappearance of the infective agent, which may reflect the natural history of the disease. Therefore, in this paper TF and TI are referred to as "active inflammatory trachoma" or "active trachoma" rather than "active infection". 
Table 1 Prevalence of trachoma by age in the Gambia, 1996

\begin{tabular}{|c|c|c|c|c|c|c|c|c|c|c|c|}
\hline \multirow[b]{2}{*}{ Age } & \multirow[b]{2}{*}{ Examined } & \multicolumn{2}{|l|}{ No signs } & \multicolumn{2}{|c|}{$T F$ and/or $T I$} & \multicolumn{2}{|l|}{$T S$} & \multicolumn{2}{|l|}{$T T$} & \multicolumn{2}{|c|}{$\mathrm{CO}$} \\
\hline & & $N$ & $\%$ & $N$ & $\%$ & $N$ & $\%$ & $N$ & $\%$ & $N$ & $\%$ \\
\hline $0-4$ & 2261 & 2112 & 93.4 & 138 & 6.1 & 4 & 0.2 & 0 & 0 & 0 & 0 \\
\hline $5-9$ & 2331 & 2176 & 93.4 & 131 & 5.6 & 23 & 1.0 & 1 & $<0.1$ & 0 & 0 \\
\hline $10-14$ & 1808 & 1742 & 96.4 & 39 & 2.2 & 35 & 1.9 & 0 & 0 & 0 & 0 \\
\hline $15-29$ & 3359 & 3192 & 95.0 & 37 & 1.1 & 128 & 3.8 & 13 & 0.4 & 3 & 0.1 \\
\hline $30-44$ & 1760 & 1591 & 90.4 & 16 & 0.9 & 153 & 8.7 & 36 & 2.0 & 7 & 0.4 \\
\hline $45+$ & 1528 & 1199 & 78.5 & 24 & 1.6 & 296 & 19.4 & 71 & 4.6 & 22 & 1.4 \\
\hline Total & 13047 & 12012 & 92.1 & 385 & 3.0 & 639 & 4.9 & 121 & 0.9 & 32 & 0.2 \\
\hline
\end{tabular}

In the first national survey in 1986 the same sampling strategy was used, although the two samples were independent. Trachoma was graded in an identical manner to the 1996 survey, again using the simplified WHO system. Interobserver studies were also undertaken to ensure correct grading with minimal variation between examiners.

\section{Results}

TRACHOMATOUS INFLAMMATION (GRADES TF OR TI)

The presence of $\mathrm{TF}$ or $\mathrm{TI}$, indicating active trachoma, was found in $3.0 \%$ of the participants (Table 1). Based on this finding, an estimated 34000 people in the Gambia had active inflammatory trachoma in 1996. Of particular interest is active trachoma among children (Table 2). The prevalence of TF or TI among children aged $0-4$ years old was $6.1 \%$ and among those aged 5-9 was 5.6\%. An estimated 12000 children aged $0-4$ and 11000 children aged 5-9 had active infection in 1996. Prevalence of active infection among children was low in Banjul City (1.2\%) and Upper River Division (1.3\%). Highest levels were found in the Lower River (11.5\%), the remainder of the Western (9.5\%) and the North Bank divisions $(7.7 \%)$.

Shown on the right in Table 2 is the distribution of prevalence of active inflammatory trachoma. Of the 73 settlements covered in the survey, $19(26 \%)$ had no cases of active infection, $24(33 \%)$ had prevalence of $1-4 \%$, $25(34 \%)$ had prevalence of $5-19 \%$, and the remaining five $(7 \%)$ had prevalence of at least $20 \%$.

Interestingly, the prevalence of active trachoma was higher in small settlements (less than 400 residents) than in large settlements $(400+$ residents). In small settlements the prevalence of active trachoma among children was $8.3 \%$ whereas in large settlements prevalence was $5.4 \%$, a 1.5 -fold variation in prevalence $(\mathrm{p}=0.002)$. This pattern was seen in all parts of the country.

Presence of active inflammatory trachoma among children was not influenced by the number of children living in a compound. Among children aged 0-9 years, prevalence of active trachoma ( $\mathrm{TF}$ or $\mathrm{TI}$ ) was $5.9 \%$ in compounds with 1-10 children, $6.3 \%$ in compounds with 11-20 children, and $5.1 \%$ in compounds with 21 or more children.

The comparison of data from the 1986 survey with the present survey provides insight into the changing pattern of active trachoma in the Gambia. (Table 3).

The national prevalence of active trachoma (TF or TI) among people aged 0-14 was $10.4 \%$ in 1986 compared with $4.9 \%$ in 1996 , a $54 \%$ reduction. The prevalence of active trachoma fell during the 10 year period in most parts of the country. Relative reductions of more than $70 \%$ occurred in Central River and Upper River divisions during the 10 year period. There was only one area where active inflammatory trachoma has increased in prevalence-the periurban area of Greater Banjul. This area has witnessed a marked population growth over the past 10 years owing to drift from the rural areas.

\section{CONJUNCTIVAL SCARRING (TS)}

Scarring was found in $4.9 \%$ of the sample (Table 1). The prevalence of trachoma scarring (TS) increased with age to a maximum of $19.4 \%$ among those aged 45 years or older. A small number of cases were found among young children.

\section{TRICHIASIS (TT), CORNEAL OPACITY (CO), AND}

BLINDNESS

The chronic sequelae of repeated infections were observed in a small proportion of participants. TT was detected in $3.3 \%$ and $\mathrm{CO}$ in $0.9 \%$ of people aged 30 years and over. TT

Table 2 Prevalence of active trachoma (TF and/or TI) by division among children aged 0-9 in the Gambia, 1996

\begin{tabular}{|c|c|c|c|c|c|c|c|c|}
\hline \multirow[b]{3}{*}{ Division } & \multirow[b]{3}{*}{ No examined } & \multirow{3}{*}{$\begin{array}{l}\text { Prevalence } \\
\text { TF/TI (\%) }\end{array}$} & \multirow{3}{*}{$\begin{array}{l}\text { No of settlements } \\
\text { examined }\end{array}$} & \multirow{3}{*}{$\begin{array}{l}\text { Prevalence range } \\
(\%)\end{array}$} & \multicolumn{4}{|c|}{ No of settlements } \\
\hline & & & & & \multicolumn{4}{|c|}{ Prevalence of active trachoma } \\
\hline & & & & & $0 \%$ & $0.1-4.9 \%$ & $5.0-19.9 \%$ & $20.0 \%+$ \\
\hline Banjul City & 170 & 1.2 & 3 & $0.0-2.0$ & 1 & 2 & 0 & 0 \\
\hline \multicolumn{9}{|l|}{ Western } \\
\hline Greater Banjul & 1280 & 5.5 & 18 & $0.0-23.4$ & 5 & 6 & 6 & 1 \\
\hline Remainder & 566 & 9.5 & 12 & $0.0-35.3$ & 1 & 3 & 7 & 1 \\
\hline Lower River & 339 & 11.5 & 6 & $0.0-19.5$ & 2 & 1 & 3 & 0 \\
\hline North Bank & 749 & 7.7 & 11 & $0.0-24.7$ & 1 & 4 & 5 & 1 \\
\hline Central River & 732 & 4.8 & 13 & $0.0-25.0$ & 3 & 5 & 3 & 2 \\
\hline Upper River & 756 & 1.3 & 10 & $0.0-8.3$ & 6 & 3 & 1 & 0 \\
\hline Total & 4592 & 5.9 & 73 & $0.0-35.3$ & 19 & 24 & 25 & 5 \\
\hline
\end{tabular}


Table 3 Prevalence of active trachoma (TF and/or TI) among children aged 0-14 in the Gambia

\begin{tabular}{|c|c|c|}
\hline Division & $\begin{array}{l}1986 \text { survey } \\
\text { prevalence } \\
(\%)\end{array}$ & $\begin{array}{l}1996 \text { survey } \\
\text { prevalence } \\
(\%)\end{array}$ \\
\hline Banjul City & 2.3 & 1.9 \\
\hline \multicolumn{3}{|l|}{ Western } \\
\hline Greater Banjul & 2.3 & 4.6 \\
\hline Remainder & 14.0 & 7.8 \\
\hline Lower River & 12.3 & 9.4 \\
\hline North Bank & 12.6 & 6.1 \\
\hline Central River & 14.1 & 4.0 \\
\hline Upper River & 5.0 & 1.2 \\
\hline Total & 10.4 & 4.9 \\
\hline
\end{tabular}

was more prevalent in women; $3.9 \%$ of women aged 30 years and over had TT, compared with $2.5 \%$ of men aged 30 years and over ( $p=0.026)$. One child under 15 had TT. Regional variation in TT prevalence was similar to that seen for active trachoma. Prevalence of TT among people aged 30 and over was lowest in Banjul City (2.4\%) and Upper River Division (1.3\%), and highest levels were found in North Bank (5.4\%) and Lower River divisions $(4.6 \%)$.

The prevalence of blinding trachomatous corneal opacity was $0.02 \%$ in 1996 . In the 1986 survey the age standardised prevalence of blinding corneal opacity was $0.10 \%$. This indicates the prevalence of blindness due to trachoma has fallen by $80 \%$ over the past decade.

Based on the survey results, there were in 1996 an estimated 10400 people with TT, 3800 with $\mathrm{CO}$, and 300 with blinding trachomatous corneal opacity in the Gambia.

\section{Discussion}

The Gambia, situated in the dry arid Sahel belt of west Africa, lies in the trachoma belt of Africa and the disease has been hyperendemic for many decades. The data presented here show trachoma to be declining. The age standardised prevalence of blinding trachomatous corneal opacities has fallen from $0.10 \%$ in 1986 to $0.02 \%$ in 1996 . Trachoma has fallen from the second leading cause of blindness in 1986 ( $17 \%$ of all blindness) to be the fifth leading cause in 1996 (5.6\% of all blindness). Similarly, the prevalence of active inflammatory trachoma among children has fallen over the past 10 years from $10.4 \%$ to $4.9 \%$.

Corneal transplantation is not currently performed in the Gambia. The decline in prevalence of blinding corneal opacities is likely to be due either to a decline in incidence through reduction of severe active infection or to reduction in the blinding complications (for example, trichiasis) through the provision of surgery. In 1986 there was an estimated backlog of 4600 people with TT requiring lid surgery, and in 1996 a backlog of 2100 . In the intervening 10 years, 1500-2000 lid operations for trichiasis have been performed. In view of the incidence of new cases of trichiasis, this is not sufficient by itself to explain the decline in the number of people with blinding complications. The reduction is also likely to be partly due to a cohort effect with the prevalence declining as many of the elderly cases die.

Primary healthcare services in the Gambia have expanded over the past decade. There is a resident village health worker in each settlement which has a population of at least 400 people. Over 1000 of these workers have received some basic health service training in primary eye care, including recognition and management of trachoma. Access to water and basic sanitation has improved during the past 10 years. By 1996 the average village had six wells, of all types, within its boundary. Access to water, defined as implying that members of the household do not spend a disproportionate part of the day fetching water, is available in up to $50 \%$ of rural areas and $97 \%$ of urban areas of the Gambia. Basic sanitation, defined in rural populations as those with adequate disposal such as pit privies, is available in $79 \%$ in rural areas while $99 \%$ of the urban population have pour-flush latrines. There has also been increased public awareness of trachoma. Two hundred schools have been screened and schoolchildren are actively encouraged to wash their faces. This has led to a knock on effect throughout the communities. In addition, all the children in 450 out of 2000 communities have been screened for trachoma.

Other communities have had dramatic reductions in prevalence of active trachoma over recent decades in the absence of a specific trachoma control programme. The reduction has been attributed to general improvements in public health measures in these communities. $^{5}$

Substantial local variation in trachoma occurrence was found in this study. Prevalence of active trachoma among children ranged from $1.2 \%$ in Banjul City to $11.5 \%$ in the Lower River Division. Of the 73 settlements examined in this study, 19 had no cases of active trachoma and 30 had prevalences of at least $5 \%$ including five communities with prevalence of at least $20 \%$. A similar pattern of variation was also seen for TT. This local variation highlights the need to include stratification in the sampling design when undertaking national or regional surveys of trachoma. Stratification enables more localised estimates of trachoma occurrence to be made.

A difference was observed between small and large settlements with higher prevalence of active inflammatory trachoma occurring in small settlements. The reasons for this may be multifactorial. People living in small settlements may have poorer access to health services. For example, there may only be one bus daily servicing a small village compared with larger settlements where there may be abundant transport and, therefore, better access to centralised health services. Large settlements also have a resident health worker and many of these local health workers have received basic training in eye care. Larger villages may have better water supplies and sanitation. These factors are likely to have 
contributed, either directly or indirectly, to the lower prevalence of active trachoma in larger settlements.

We expect trachoma to continue to decline as a public health problem with fewer cases of trachoma blindness in the near future. We anticipate the prevalence of active infection to further fall in the coming decade. Despite the rapid increase in the population of the Gambia, the absolute number of people with active trachoma is also expected to decline. In the meantime, prompt surgery of existing cases of $\mathrm{TT}$ is required to prevent these cases from progressing to blindness.
This study was funded by SightSavers International and the British Council for the Prevention of Blindness. We thank the government of the Gambia for its support.

1 Faal H, Minassian D, Sowa S, et al. National survey of blindness and low vision in the Gambia. $\mathrm{Br} F$ Ophthalmol 1989,73

2 Faal H, Dolin PJ, Ajewole J, et al. Prevalence and causes of blindness and low vision in the Gambia. Brf Ophthalmol (in press)

3 Thylefors B, Dawson CR, Jones BR, et al. A simplified system for the assessment of trachoma and its complications. Bull World Health Organ 1987;65:477-83.

4 Bailey RL, Hampton TJ, Hayes LJ, et al. Polymerase chain reaction for the detection of ocular chlamydial infection in trachoma endemic communities. F Infect Dis 1994;170: 709-12.

5 Dolin PJ, Faal H, Johnson GJ, et al. Reduction of trachoma in a sub-Saharan village in absence of a disease control programme. Lancet 1997;349:1511-12. 